\title{
Through-Thickness Characterization of Microstructure and Texture in High Purity Aluminum Processed to High Strain by Accumulative Roll-Bonding
}

\author{
Naoya Kamikawa ${ }^{1, * 1}$, Nobuhiro Tsuji ${ }^{1, * 2}$, Xiaoxu Huang ${ }^{2}$ and Niels Hansen ${ }^{2}$ \\ ${ }^{1}$ Department of Adaptive Machine Systems, Graduate School of Engineering, Osaka University, Suita 565-0871, Japan \\ ${ }^{2}$ Center for Fundamental Research: Metal Structures in Four Dimensions, Materials Research Department, \\ Ris $\varnothing$ National Laboratory, DK-4000 Roskilde, Denmark
}

The deformation microstructure and texture have been characterized by electron backscatter diffraction (EBSD) and transmission electron microscopy (TEM) through the thickness of high purity (99.99\% purity) aluminum sheets processed by accumulative roll-bonding (ARB) up to a total strain of 4.8. This processing route creates a complex strain path, resulting in a characteristic texture changes through the thickness of the sample, very different from that obtained by conventional rolling, and also in a randomization of the overall deformation texture. The microstructure is composed of an equiaxed structure with a high concentration of high-angle boundaries and a small fraction of a lamellar structure with a high concentration of low-angle boundaries. The observations showed a coupling between the local texture and microstructure; the equiaxed regions are composed of deformation texture components and random texture components while the lamellar regions are composed of the deformation texture components, i.e. rolling or shear texture components. [doi:10.2320/matertrans.MA200702]

(Received January 11, 2007; Accepted March 26, 2007; Published July 25, 2007)

Keywords: accumulative roll-bonding (ARB), ultrafine grains, high purity aluminum, microstructure, local texture

\section{Introduction}

Mechanical properties of polycrystalline metals and alloys are strongly dependent on the grain size, and a decrease in grain size generally leads to an increase both in the strength and toughness without impairment of ductility. ${ }^{1)}$ An extrapolation of this behavior to fine grain sizes should therefore result in a significant improvement of strength, toughness and ductility in ultrafine grained materials with an average grain size of less than $1 \mu \mathrm{m}$. Therefore, grain refinement processes has been a subject of extensive research for the last decade..$^{2-4)}$

High strain deformation is a promising method to develop ultrafine grained structures in bulk metals, for example, high pressure torsion (HPT) ${ }^{5}{ }^{5}$ equal channel angular extrusion (ECAE) ${ }^{6,7)}$ cyclic extrusion compression $(\mathrm{CEC})^{8)}$ and accumulative roll-bonding (ARB) ${ }^{9)}$ Among these processes, ARB may be easily applicable to industrial production since the basic process is rolling.

In previous studies, $\mathrm{ARB}$ has been applied to a large number of metals and alloys, and the results have demonstrated that a dramatic enhancement in strength, in many cases 2 to 4 times higher than those of coarse grained specimens, can be achieved. ${ }^{3,10)}$ On the other hand, it has been also found that the ARB processed specimens show limited ductility, typically less than $10 \%$ of total elongation in uniaxial tension, ${ }^{11,12)}$ so that an optimized combination of strength and ductility by annealing after deformation may be required. However, a heterogeneous structural coarsening and conventional recrystallization take place during annealing especially at high temperatures, ${ }^{13}$ ) related to the high stored energy and structural heterogeneity in the deformed specimens. In order to understand this behavior of materials

\footnotetext{
${ }^{* 1}$ Present address: Materials Research Department, Risø National Laboratory, Denmark

${ }^{* 2}$ Corresponding author, E-mail: tsuji@ams.eng.osaka-u.ac.jp
}

produced by ARB, the heterogeneity of both the structure and the texture of the materials must be investigated in detail, which is the objective of the present study of pure aluminum (99.99\% purity) deformed up to a strain of 4.8 .

\section{Experimental}

High purity aluminum (99.99 mass\% Al-0.003 Si-0.003 $\mathrm{Fe}-0.001 \mathrm{Cu}$ ) in the form of a cast ingot was cold-rolled to $1 \mathrm{~mm}$ thickness and annealed at $350^{\circ} \mathrm{C}$ for $0.5 \mathrm{~h}$ to obtain a fully recrystallized structure with an average grain size of $41 \mu \mathrm{m}$. The annealed sheets were cut into dimensions of $1 \mathrm{~mm}$ thickness, $40 \mathrm{~mm}$ width and $250 \mathrm{~mm}$ length, as starting samples, and ARB was applied to achieve high strain deformation. The principle and detailed procedure of the ARB process have been described previously.,10) After degreasing and wire-brushing, two sheets of the starting material were stacked and roll-bonded at room temperature under dry conditions without using any lubricant. A two-high rolling mill with rolls of $310 \mathrm{~mm}$ diameter was used for this roll-bonding. The roll bonding was carried out by a thickness reduction of $50 \%$ in one pass at a peripheral speed of $17.5 \mathrm{~m}$ minute $e^{-1}$, corresponding to a nominal strain rate of $19 \mathrm{~s}^{-1}$. The roll-bonded sheet was immediately water-cooled after rolling to reduce structural changes caused by the rollinginduced temperature increase. The roll-bonded sheet was cut into half length and used for the next ARB cycle without changing the rolling direction (RD). The procedure of cutting, stacking and roll-bonding was repeated for up to 6 cycles, corresponding to a total thickness reduction of $98.4 \%$, or an total equivalent strain of 4.8. Note that this equivalent strain can be calculated from an equation of $(2 / \sqrt{3}) \ln \{1 /$ $(1-r)\}$ where $r$ is a total thickness reduction, assuming that the rolling deformation is plane-strain compression. In reality, however, a non-lubricated rolling as used in the present study leads to a redundant shear strain due to the friction between the sheet and the rolls. As a result, the strain 


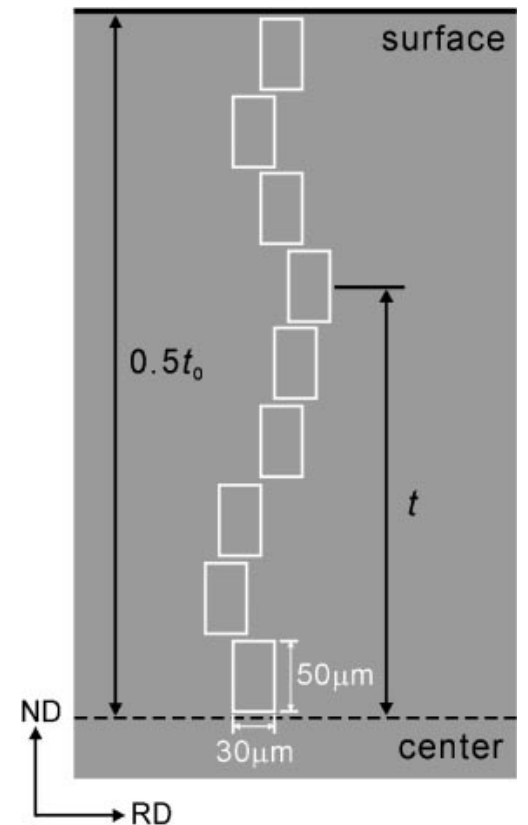

Fig. 1 Schematic illustration showing the EBSD scanned areas. The rectangular areas of $50 \mu \mathrm{m}$ in $\mathrm{ND}$ and $30 \mu \mathrm{m}$ in RD were scanned throughout the thickness. The thickness location of each scanned area was defined as $t / t_{0}$, where $t_{0}$ is the total thickness of the sheet and $t$ is the distance from the thickness center to the center of the rectangular area.

is very high near the surface, and the strain distribution through the thickness will be heterogeneous.

The deformation structure and texture of the ARB processed specimens were characterized by electron backscatter diffraction (EBSD) and transmission electron microscopy (TEM). Thin foils parallel to the longitudinal section, including the normal direction (ND) and RD of the deformed sheets, were prepared by electropolishing at $-15^{\circ} \mathrm{C}$ and at a voltage of $20 \mathrm{~V}$ in a solution of $300 \mathrm{ml}$ $\mathrm{HNO}_{3}+700 \mathrm{ml} \mathrm{CH}_{3} \mathrm{OH}$. Orientation mapping by the EBSD technique was carried out in FEI XL30S SEM with a field emission gun operated at $15 \mathrm{kV}$. TSL orientation image microscopic (OIM) programs were used for the EBSD measurements and analyses. The EBSD scans were carried out at different thickness locations from the center to the surface of the 6-cycle ARB processed sheet to examine the structure and texture heterogeneities, as schematically shown in Fig. 1. Nine rectangular areas, each having a size of $50 \mu \mathrm{m}$ in ND and $30 \mu \mathrm{m}$ in RD, were scanned at a step size of $100 \mathrm{~nm}$. The thickness location of each scanned area was defined as $t / t_{0}$, where $t_{0}$ is the total thickness of the sheet and $t$ is the distance from the sheet center to the central point of the scanned rectangular area. For example, the center, quarter thickness and surface of the sheet can be described as $t / t_{0}=0,0.25$ and 0.5 , respectively. An online Kikuchi-line analysis technique $^{14)}$ in JEOL 2000FX TEM operated at $200 \mathrm{kV}$ was used for fine-scale orientation measurements.

\section{Results}

Figure 2 shows the EBSD maps, including ND color map, $\mathrm{RD}$ color map and grain boundary (GB) map, obtained from the through-thickness measurement for the 6-cycle ARB processed specimen. The ND and RD maps present the crystallographic direction parallel to the ND and RD of the sheet, respectively, using the color code shown in the standard stereographic triangle. In the GB maps, high-angle grain boundaries with misorientation angles greater than $15^{\circ}$ are shown as green lines while low-angle grain boundaries between 2 and $15^{\circ}$ as red lines. Boundaries less than $2^{\circ}$ are not included in the analysis due to the uncertainty in the determination of low-angle boundaries by EBSD. ${ }^{15,16)}$ In order to remove unreliable data points, a clean-up for the raw scanned data was carried out by the grain dilation method in the TSL software. In this method, the orientations of isolated data points, composed of only one data point, were changed into the orientation of the majority of the surrounding points. This procedure removed mainly isolated data points detected in the vicinity of boundaries. The thickness location of the scanned area, $t / t_{0}$, is also indicated in the figure. The ND and $\mathrm{RD}$ maps consist of a large variety of colored regions, indicating the existence of many different orientations in each scanned region. It is also seen that the orientation distribution in each map varies from the thickness center to the surface of the sheet. The GB maps demonstrate a high density of high-angle boundaries in each region, corresponding to the color distributions in the ND and RD maps. The majority of the structure observed in this specimen consists of crystallites that are slightly elongated to the RD and almost equiaxed. These crystallites are surrounded mainly by highangle boundaries. At the same time, a small fraction of lamellar crystallites elongated in the RD with a high concentration of low-angle boundaries is also observed, as indicated by arrows in the figure. Hereafter, the regions consisting of slightly elongated crystallites and lamellar crystallites will be termed equiaxed structure (region) and lamellar structure (region), respectively. It can be seen that the boundary spacing of the equiaxed structure is larger than that of the lamellar structure.

Figure 3(a)-(d) shows some examples of the $\{111\}$ pole figures obtained from the EBSD analysis to demonstrate a texture inhomogeneity through the thickness. The thickness locations from which the pole figures were obtained are indicated. Ideal orientations of $\{112\}\langle 111\rangle$ (Copper), $\{123\}\langle 634\rangle$ (S) and $\{001\}\langle 110\rangle$ (Rotated Cube) components are plotted in the figures, where only one variant is shown for Copper and $\mathrm{S}$ in order to avoid the complication of the figures. In the near-center layers (Fig. 3(a), (b)), Copper and $\mathrm{S}$ orientations, typical rolling texture components in pure fcc metals with relatively high stacking fault energy, are observed. ${ }^{17-20)}$ The intensity of the rolling texture is weaker in the layer closest to the thickness center $\left(t / t_{0}=0.03\right.$, Fig. 3(a)) than in the internal layer $\left(t / t_{0}=0.09\right.$, Fig. 3(b)). On the other hand, the texture near the surface (Fig. 3(c), (d)) is a Rotated Cube texture and a minor ND // $\langle 111\rangle$ fiber, which are known as surface shear texture components developed by high-frictional rolling. ${ }^{21-23)}$ The intensity of this shear texture $\left(t / t_{0}=0.46\right.$, Fig. $\left.3(d)\right)$ is weaker than in the subsurface layer $\left(t / t_{0}=0.30\right.$, Fig. 3(c)). This specimen reveals a heterogeneous orientation distribution from the thickness center to the surface of the sheet. However, it can be seen from the pole figure obtained from the sum of EBSD data through the thickness (Fig. 3(e)) that the overall texture 

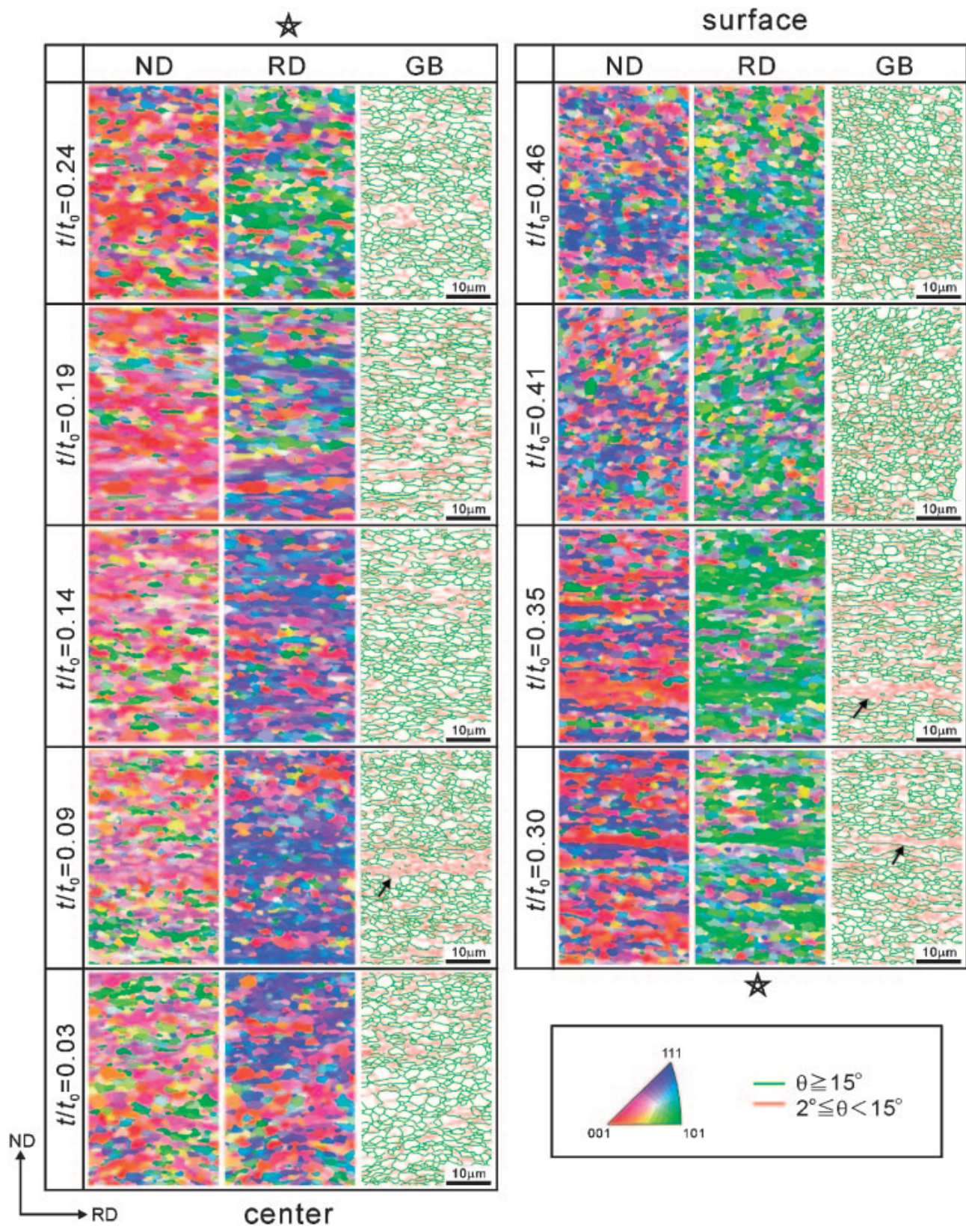

Fig. 2 EBSD maps (ND map, RD map and GB map) through thickness of the deformed specimen.

is weak compared with a pure aluminum conventionally cold-rolled to high strain. ${ }^{17)}$

The spatial distributions of individual deformation texture components were analyzed from the EBSD data and are shown in Fig. 4. In this analysis, $\{112\}\langle 111\rangle$ (Copper), $\{123\}\langle 634\rangle(\mathrm{S})$ and $\{110\}\langle 112\rangle$ (Brass) were classified as rolling texture components while $\{001\}\langle 110\rangle$ (Rotated Cube), $\{111\}\langle 112\rangle$ and $\{111\}\langle 110\rangle$ were classified as shear texture components, and $15^{\circ}$ spread from the ideal orientation was used for the classification. Other orientations that do not belong to either rolling or shear texture were referred to as random texture components, and are shown in white. Black and gray lines indicate high-angle and low-angle boundaries, respectively. In the near-center layers at the thickness location of $t / t_{0}=0.03,0.09$ and 0.14 , the equiaxed regions are characterized by randomly mixed rolling texture and random texture orientations, resulting in a high concentration of high-angle boundaries. On the other hand, the elongated lamellar regions represent rolling texture components. For example, a high concentration of low-angle boundaries indicated by an arrow in Fig. $2\left(t / t_{0}=0.09\right)$ represent a Copper texture component. As the thickness location is closer to the surface, the fraction of rolling texture components decreases and that of shear texture components increases. In the layers near the thickness quarter $\left(t / t_{0}=0.19,0.24\right)$, a large amount of crystallites with Rotated Cube orientations can be observed. At the thickness location of $t / t_{0}=0.30$ and 0.35 , shear texture and random texture components are distributed randomly in the equiaxed region while the lamellar regions surrounded by low-angle boundaries consist of one of the shear texture components. The regions indicated by arrows form Rotated Cube texture bands. In the nearsurface layers $\left(t / t_{0}=0.41\right.$ and 0.46$)$, the majority of the structure is equiaxed, and three shear texture components and 
(a) $t / t_{0}=0.03$

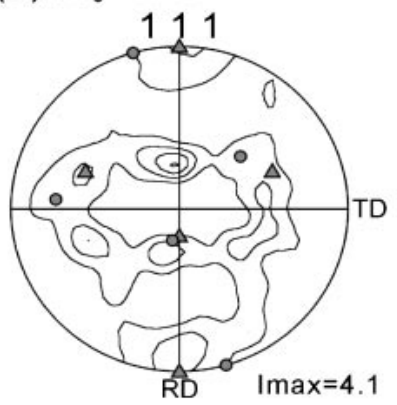

(c) $t / t_{0}=0.30$

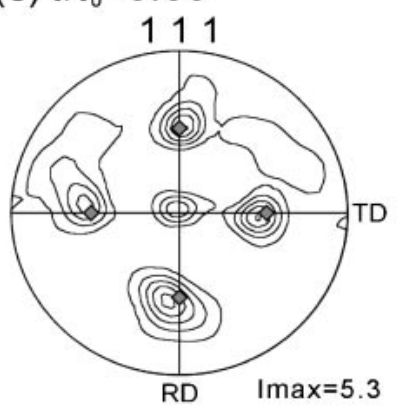

(b) $t / t_{0}=0.09$

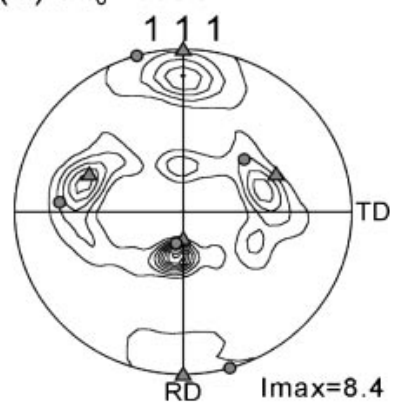

(d) $t / t_{0}=0.46$

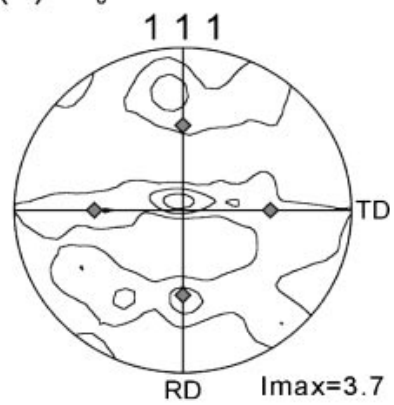

(e) total thickness

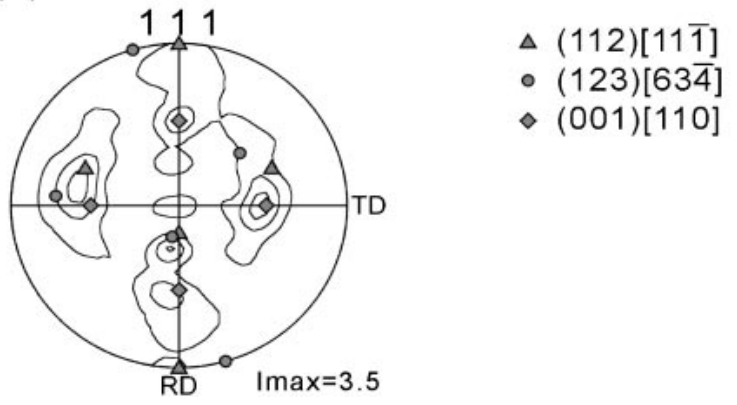

Fig. $3\{111\}$ pole figures constructed from the EBSD data of the deformed specimen. The pole figures ( $\mathrm{a}, \mathrm{b}, \mathrm{c}$ and $\mathrm{d}$ ) correspond to the thickness location of $t / t_{0}=0.03,0.09,0.30$ and 0.46 in Fig. 2, respectively, and (e) were obtained by sum of nine different areas through the thickness. Contour levels (x random): 1.0, 2.0, 3.0, .., 8.0.

random texture components are distributed randomly. In summary, a general trend is that the equiaxed regions with a high concentration of high-angle boundaries have a random mixture of deformation texture components and random components while the lamellar regions with a high concentration of low-angle boundaries are oriented toward one of the deformation texture components. This indicates a correlation between local texture and microstructure, ${ }^{13,24)}$ which will be discussed later.

Figures 5(a) and (b) show the area fractions of individual rolling texture components and shear texture components, respectively, plotted as a function of $t / t_{0}$. The total fraction of rolling and shear texture components is shown in Fig. 5(c). The center layers are composed of Copper and $\mathrm{S}$ orientations with a small fraction of the Brass orientation. The fraction of shear texture components is quite low near the thickness center. With increasing $t / t_{0}$ (approaching the surface), the fraction of rolling texture components decreases and the fraction of shear texture components increases, changing to a Rotated Cube orientation and minor orientations of

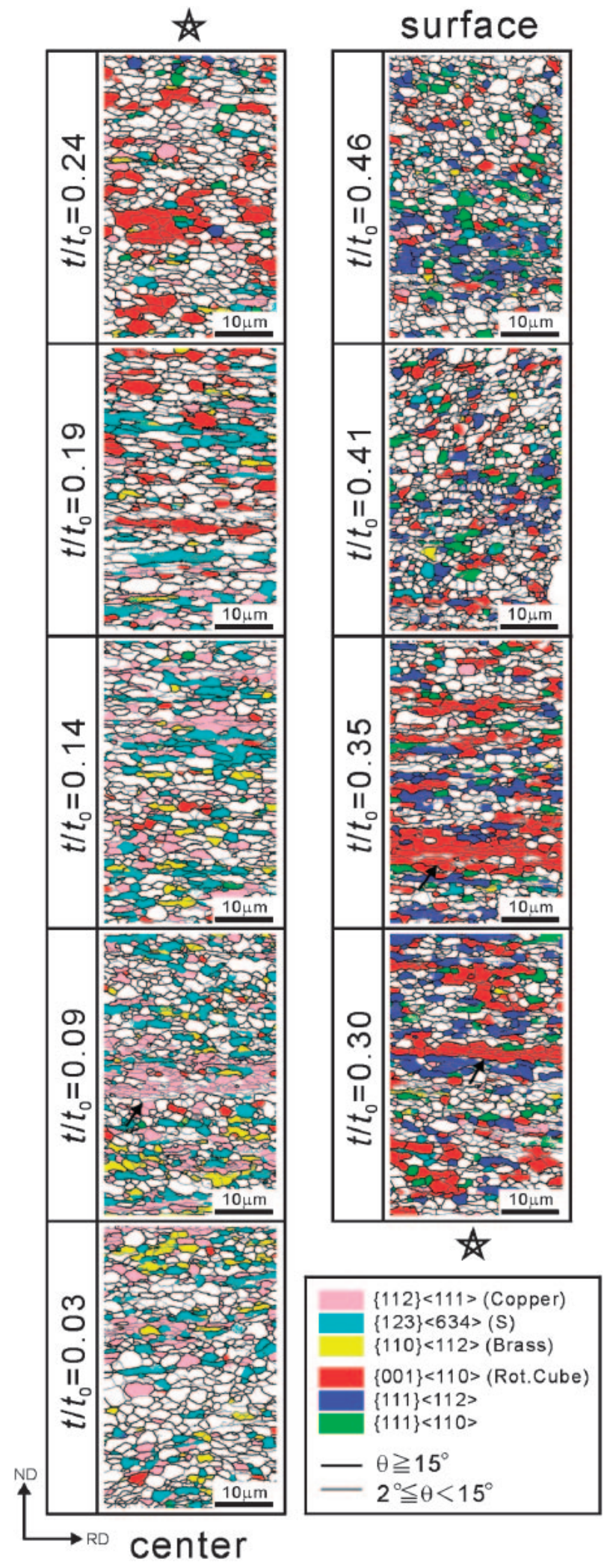

Fig. 4 Texture component maps through thickness of the deformed specimen.

$\{111\}\langle 112\rangle$ and $\{111\}\langle 110\rangle$ in the near-surface layers. Similar texture distributions throughout the thickness of a commercial purity aluminum (AA1100, 97.5\% purity) and an Al-1.3 Fe-0.09 Si alloy (AA8079) deformed by ARB up to a strain of $\sim 10$ have been reported. ${ }^{25,26)}$ The total fraction of rolling 

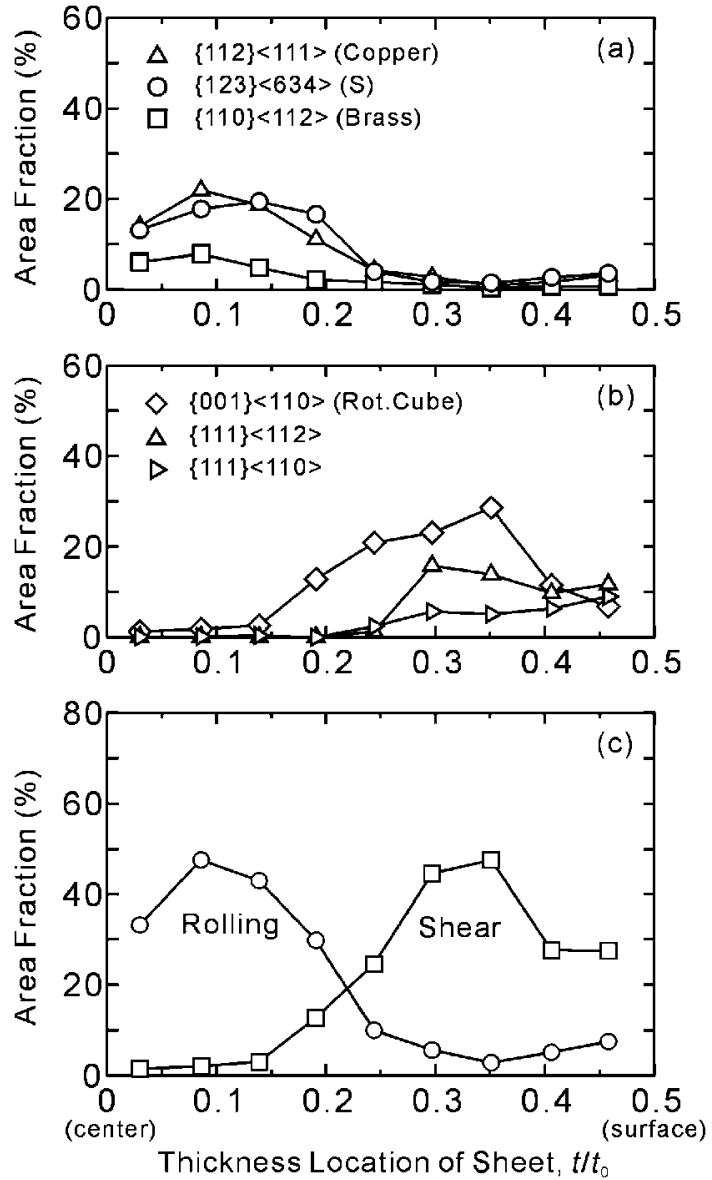

Fig. 5 Area fractions of individual texture orientations through thickness of the deformed specimen. The fractions were determined from the EBSD data. (a) Rolling texture orientations, (b) shear texture orientations and (c) sum of rolling or shear texture orientations.

texture components is stronger than that of the shear texture between the thickness center and $t / t_{0}=0.225$, while the opposite tendency can be seen between $t / t_{0}=0.225$ and the surface. It is interesting that the total fraction of rolling texture components shows a local minimum in the center layer and a maximum around $t / t_{0}=0.10$, while the total fraction of shear texture components shows a local minimum in the surface layer and a maximum at the thickness location of $t / t_{0} \sim 0.35$. It should also be emphasized that the total fraction of rolling texture components is less than $50 \%$ in each layer, which is significantly smaller than that in an Al$0.007 \mathrm{Fe}$ conventionally cold-rolled up to $99.5 \%$ where the rolling texture fraction is about $75 \% .^{19)}$

Figure 6 shows the average boundary spacing in the ND $\left(d_{t}^{\mathrm{ALL}}\right)$, fraction of high-angle boundaries $\left(f_{\mathrm{HAGB}}\right)$ and average misorientation of the boundaries $\left(\theta_{a v}\right)$ analyzed from the EBSD data, as a function of $t / t_{0}$. The $d_{t}^{\mathrm{ALL}}$ was determined by an intercept counting method, taking account of all boundaries with misorientations of $2^{\circ}$ and above. The $f_{\mathrm{HAGB}}$ and $\theta_{a v}$ were calculated from all the boundaries. It is obvious from Fig. 6(a) that the boundary spacing is almost constant at around $1 \mu \mathrm{m}$. This corresponds well with the microstructural homogeneity reported in the various kinds of ARB processed sheets. ${ }^{27,28)}$ In Fig. 6(b), the fraction of highangle boundaries and the average misorientation also show
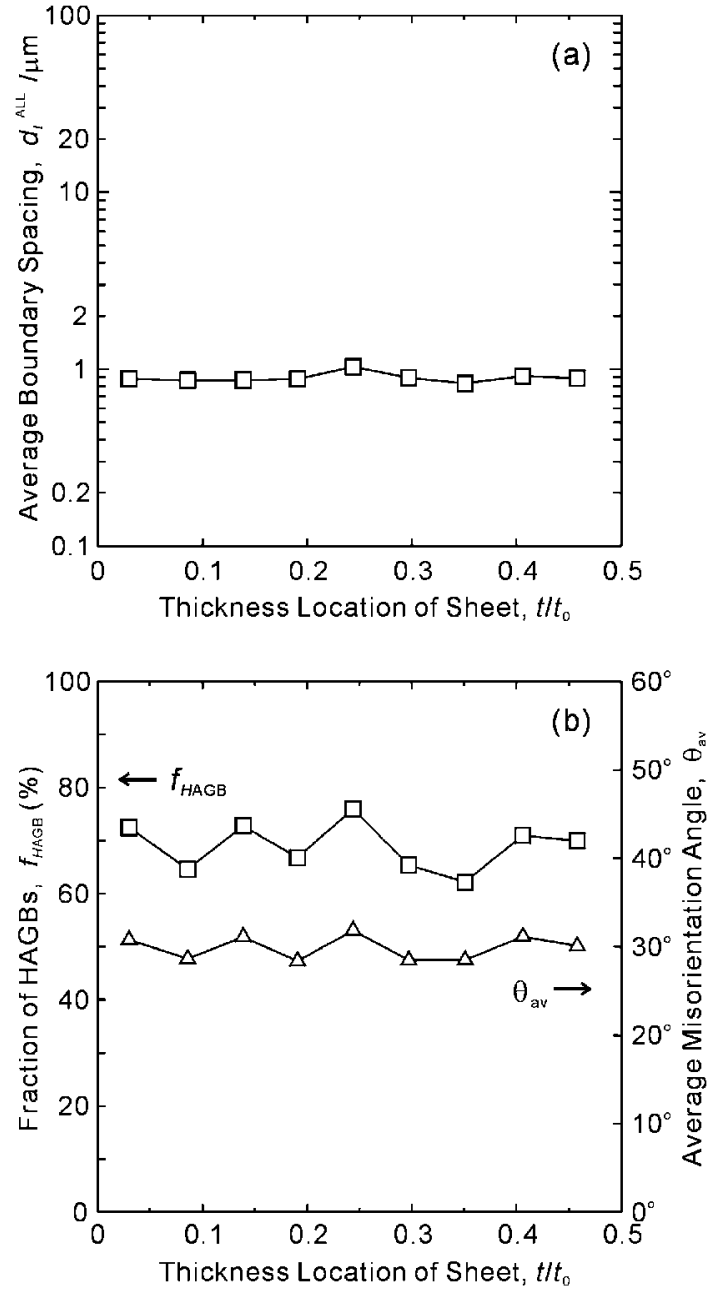

Fig. 6 Microstructural parameters through thickness of the deformed specimen. The parameters were determined from the EBSD data.

relatively homogeneous distributions, but it is also found that the data points at the thickness location of $t / t_{0}=0.09,0.30$ and 0.35 reveal lower values than other data points, which may be due to the high concentration of low-angle boundaries in the lamellar bands observed in these layers, as shown in Figs. 2 and 4. It can be summarized based on Fig. 6 that the structural distribution is fairly homogeneous in terms of the boundary spacing distribution, but rather heterogeneous in terms of the boundary misorientation distribution depending on the distributions of texture components in the specimen.

\section{Discussion}

This study demonstrated that a high purity aluminum sample deformed by 6 cycles of ARB revealed the following characteristic textural features.

(i) The near-center layers had a mixture of rolling texture components (Copper, S and Brass) and random texture components, while the near-surface layers showed a mixture of shear texture components (Rotated Cube and $\mathrm{ND} / /\langle 111\rangle$ ) and random texture components.

(ii) The overall texture was relatively weak even though high strain deformation had been imposed on the specimen. 


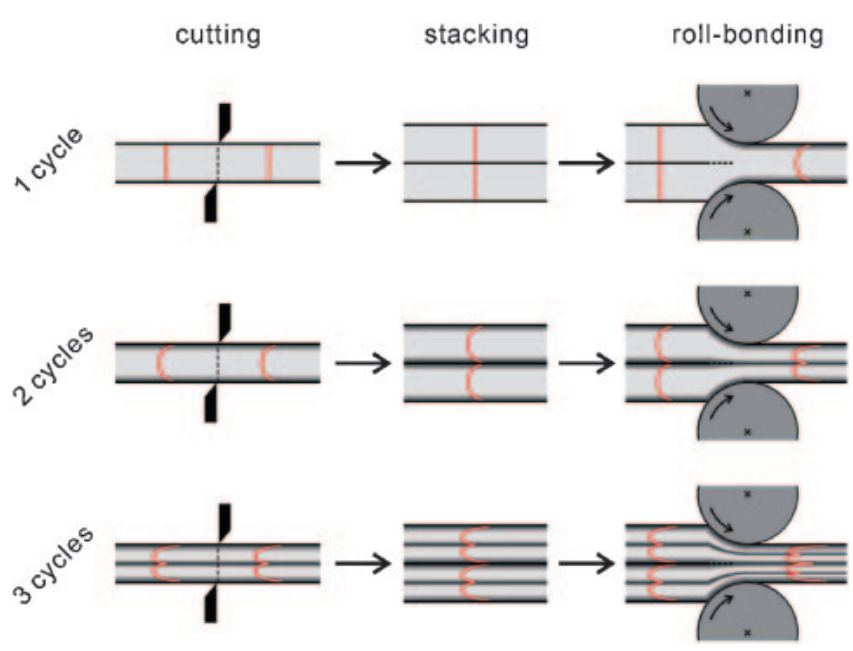

Fig. 7 Schematic illustration showing the distribution of the redundant shear strain through thickness of ARB processed sheet.

(iii) Local minima of rolling texture components at the sheet center and of shear texture components at the surface were observed.

Such textural features may be strongly related to a specific deformation geometry in the ARB process, which will be discussed in the next section.

\subsection{Deformation geometry in the ARB process}

In the present study, the ARB process was carried out under dry conditions without any lubricant. In this case, due to the high friction between the sample and the rolls, a large amount of redundant shear deformation, in addition to plane strain compression, will be introduced. The strain distribution through the thickness will therefore be heterogeneous. Especially a strong shear texture will be present at or near the surface. This phenomenon is well known for high friction rolling, e.g. hot rolling. ${ }^{29,30)}$ In the ARB process, characteristically, a repetition of cutting, stacking and roll-bonding is carried out for each ARB cycle, making the situation more complicated. To illustrate this effect of the number of cycles, Figure 7 shows the distribution of the shear strain up to 3 cycles, and Figure 8 shows the expected shear strain distributions up to 6 cycles of ARB, as a function of $t / t_{0}$. In Fig. 7, red lines indicate the change in the distribution of redundant shear strain, and dark-gray regions indicate the severely sheared layers near the surface. After 1 cycle of $\mathrm{ARB}$, the upper and lower sheet surface is sheared due to the high friction, and a parabolic distribution of shear strain symmetrical to the mid-thickness plane is introduced in the specimen (see Fig. 8(a)), as has previously been reported by Lee et $a l .{ }^{31)}$ In the second cycle of ARB, because of cutting and stacking, two sheet surfaces in the first ARB cycle is moved to the thickness center and the roll-bonding process introduces an additional parabolic shear strain, leading to a double parabolic shear strain distribution (Fig. 8(b)). This procedure is repeated during each ARB cycle, so that the expected shear strain distribution through the thickness becomes more and more complicated with increasing number of ARB cycles, as shown in Fig. 8(c), (d). This complex strain distribution, or strain path may be the underlying cause
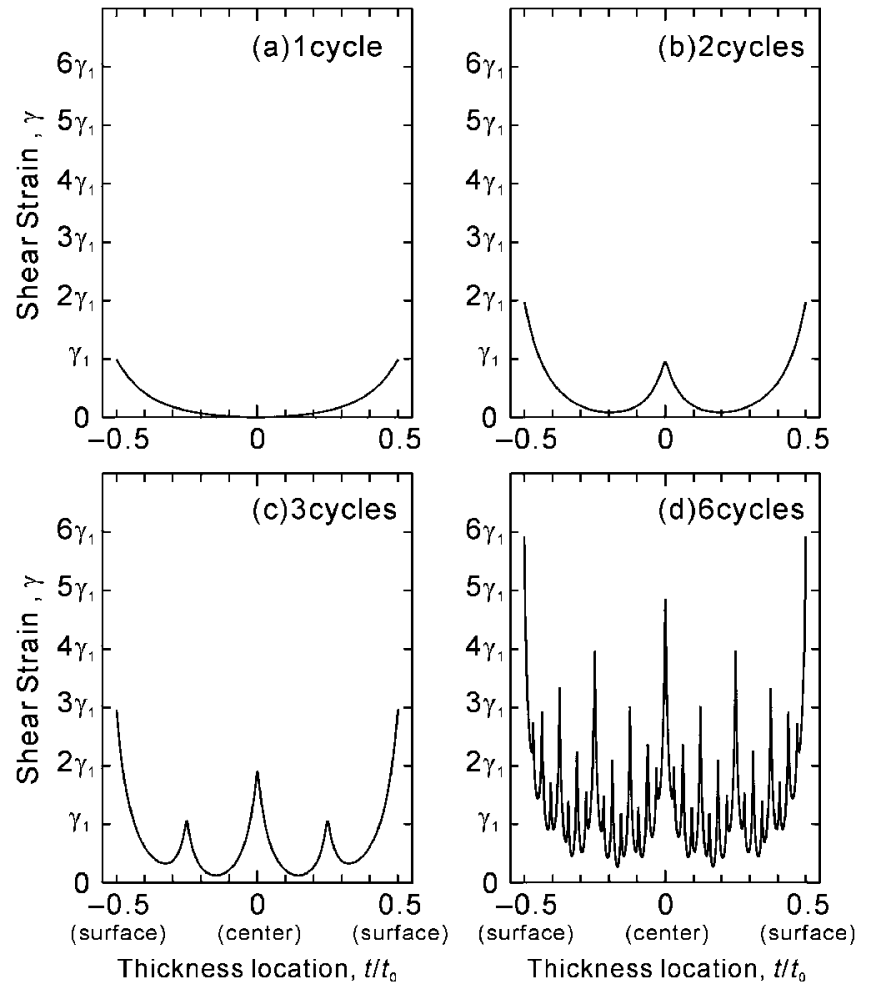

Fig. 8 Expected shear strain distribution through thickness of ARB processed sheet.

for the variation in texture through the thickness of the ARB processed sheet.

\subsection{Texture inhomogeneity in ARB processed specimen}

The expected shear strain distribution in the 6-cycle ARB specimen reveals many local peaks of redundant shear strain, as shown in Fig. 8(d), but the observation (Fig. 5(c)) shows relatively smooth changes in local texture and does not follow the expected pattern of accumulated shear strain. For example, since the center layer in the last cycle has been the surface layer in the previous cycle, the center layer should show a texture reflecting a shear strain peak. However, a weak rolling texture was observed at the thickness center. This result indicates that a shear texture developed in the surface layer of the sheet is easily destroyed by a plane-strain compression (pure rolling with no shear) imposed in the center layer in the following ARB cycle. Similar observations that plane strain compression can rapidly destroy the shear texture have been reported in previous studies. ${ }^{26,28,32)}$ For confirmation, a through-thickness EBSD mapping was carried out for a 7-cycle ARB processed specimen, and it was found that the orientation distribution through the thickness of the 7-cycle specimen was almost identical to that observed in the 6-cycle specimen (Figs. 4 and 5). This shows that the shear texture in the surface layer of the 6-cycle ARB specimen was destroyed and rotated into a rolling texture in the near-center layer in the following ( 7 th) cycle of $\mathrm{ARB}$. It is also found that the center layer in the 6-cycle ARB specimen, which moved into the quarter thickness layer in the 7th ARB cycles, now had developed a shear texture, i.e. a rolling texture was rotates toward a shear texture where the next cycle changes the strain path. 
In previous studies, computer simulations for texture prediction based on a full-constraints Taylor model by Heason and Prangnell ${ }^{25,26)}$ have demonstrated that the Rotated Cube orientation can be rapidly rotated toward the Copper orientation by additional plane strain compression (50\% rolling). This simulation supports the results obtained in the present study. On the other hand, it has been also claimed that the gradual rotation of Copper, $\mathrm{S}$ and Brass rolling texture orientations toward the Rotated Cube orientation and other minor texture orientations may occur by shear deformation. ${ }^{25,26)}$ This is not in good agreement with the present result, showing that the rolling texture components were switched into shear texture components by a single cycle of ARB when they experienced the shear deformation by moving from the center to the quarter thickness location. Note that in the present study the ARB process was carried out using high friction rolling, so that the magnitude of the shear strain imposed by each cycle of ARB may be very high. The magnitude of the shear strain has been experimentally estimated by Lee et al., ${ }^{31)}$ and as an example it was found that a shear strain of $\sim 8$ can be introduced at the surface $\left(t / t_{0}=\right.$ 0.5 ) and a strain of $\sim 3.4$ at $t / t_{0}=0.4$ after a single cycle of $\mathrm{ARB}$ in a commercial purity aluminum. Therefore, the rapid rotation of the rolling texture toward the shear texture after a subsequent roll-bonding step may not surprising. The cyclic process of texture transition from shear to rolling texture and vice versa may take place during the ARB cycles, which may retard a development of strong texture and lead to a relatively weak deformation texture and a characteristic distribution of different texture components through the sample thickness. In other words, the strain path change may be effective in randomizing the deformation texture.

It is somewhat surprising that the surface layer of the 6cycle ARB specimen showed a local minimum of shear texture even though this layer should have a maximum accumulation of shear strain (Fig. 5(c)) to be discussed in the following. Sakai et al. ${ }^{29,30,33)}$ have reported a similar observation in hot rolled sheets both in bcc and fcc steels, where a shear texture did not show a maximum in the surface layer but in an inner layer near the surface. In these cases, ${ }^{29,30,33)}$ the sheet samples were heated to high temperatures before rolling but the rolls were kept at room temperature, so that the sheet surfaces were cooled by the rolls during deformation. As a result, the flow stress, or deformation resistance, in the surface layers was higher than that in the inner layers, which may reduce the shear deformation and the shear texture at the surface. However, this interpretation may not be applied the present study, since the rolling was carried out at room temperature. Another possibility is sticking of the surface during high friction rolling, as an adhesion zone near the surface restricts the sample deformation due to a sticking between the sheet surface and the rolls. ${ }^{34)}$ A third possibility is that a reverse shear deformation within the roll bite during rolling may lead to the decrease in shear texture evolution at the surface. This relates to the existence of a neutral point in rolling where the speed of the rolls and the sheet surface is identical within the roll bite. ${ }^{35)}$ On the entrance side of the roll bite before the neutral point, the surface of the sheet is sheared toward the rolling direction, as the speed of the rolls is faster than that of the sheet surface. By contrast, on the exit side of the roll bite after the neutral point, the speed of the sheet surface is faster than that of the rolls, so that the shear direction at the surface is opposite to the rolling direction. Therefore, the layer sheared in the rolling direction before the neutral point will experience a reverse shear deformation in the opposite direction after the neutral point. This reverse shear deformation can be considered as a type of strain path change before and after the neutral point, which may play a significant role in texture randomization and reduce the shear texture evolution in the surface layer as shown in Fig. 5(c). Assuming that the reverse shear deformation would be concentrated in the surface layer since there is only a small thickness change from the neutral point to the exit of the roll bite, it is reasonable that the inner layers at the thickness location of $t / t_{0}=0.30 \sim 0.35$ showed a maximum shear texture because these layers may not have undergone a reverse shear deformation. This discussion may also explain why the center layer showed a local minimum of rolling texture in the last ARB cycle (Fig. 5(c)).

In concluding this discussion of heterogeneities through the thickness of rolled sheet, previous investigations ${ }^{22,23,36,37)}$ have shown an texture gradient in cold-rolled fcc metals and demonstrated that a shear texture was observed in intermediate layers, as also observed in the present study. However, in these previous investigations ${ }^{22,23,36,37)}$ the rolling process was carried out with a geometrical factor of $h_{m} / L$ larger than 2, where $h_{m}$ is the mean sheet thickness during rolling and $L$ is the contact length between the sheet and the roll, leading to a geometrical shear concentration in the quarter thickness layer. However, in the present work as in other studies ${ }^{29,30,33)}$ rolling with $h_{m} / L$ much smaller than 2 have been used, leading to a domination of frictional shear and development of a shear texture in the near-surface layer.

\subsection{Local texture, structural morphology and misorien- tation distribution}

An important finding is the correlation between the local texture and the microstructure. Two different structural morphologies were observed in the ARB processed specimen; one is a relatively equiaxed structure and the other a lamellar structure with a pronounced elongation in the RD. It was found that the equiaxed crystallites were mainly surrounded by high-angle boundaries due to random distribution of deformation texture components and random texture components while the lamellar structure was subdivided by low-angle boundaries and each local area of lamellar structure represented one deformation texture component. Considering the area fraction of these two structural types, the fraction of the lamellar structure decreases with increasing strain and appears as isolated areas in the final microstructure (see Fig. 2) A similar mixed structure has also been observed by Kikuchi-line analysis in the TEM. ${ }^{13,24)}$ Moreover, the TEM observations show that the equiaxed region had a lower interior dislocation density than the lamellar region. ${ }^{13)}$ These results indicate that the local texture may affect the structural morphology and recovery behavior. In general, high-angle boundaries have higher migration mobility than low-angle boundaries, and regions containing many high-angle boundaries may therefore migrate faster 
than regions with a high density of low-angle boundaries. High-angle boundaries may also act as sinks for dislocation annihilation, underpinning the observation ${ }^{13)}$ that the dislocation density is low in the equiaxed structure. Note that in the present study, because of the high purity of the material, recovery and boundary migration may take place during or just after deformation.

\section{Conclusions}

(1) The ARB specimen showed characteristic textural features through the sheet thickness, but the overall texture was relatively weak. This result had been related to the complicated strain path during $\mathrm{ARB}$, and it was shown that the orientation distribution observed in the ARB processed specimen did not follow the accumulation of redundant shear strain introduced during the ARB cycles.

(2) It was observed that the shear texture components formed at the surface might be rapidly destroyed during the following cycle and switched into rolling texture components near the center. It was also found that rolling texture components could be switched into shear texture components within an intermediate layers due to a shift in position, changing the deformation from rolling to shear. Such changes in strain path may reduce the strength of the deformation texture and create a characteristic texture through the sheet thickness.

(3) The deformed specimen had two different structural morphologies. The majority of the structure was composed of a relatively equiaxed structure with a high concentration of high-angle boundaries, while a small fraction showed an elongated lamellar structure with a high concentration of low-angle boundaries. A good correlation between the local texture and structure was found, as the equiaxed regions had a mixture of deformation texture components and random texture components while the lamellar regions each represented one of the deformation texture components.

\section{Acknowledgement}

The present study was supported by the 21 st Century COE program, the Center of Excellence for Advanced Structural and Functional Materials Design at Osaka University, funded by the Ministry of Education, Culture, Sports, Science and Technology of Japan. XH and NH also acknowledge the Danish National Research Foundation for supporting the Center for Fundamental Research: Metal Structures in Four Dimensions at Ris $\varnothing$ National Laboratory, within which part of the present work was performed. The authors thank Drs. G. Winther, D. Juul Jensen, O. V. Mishin and Prof. B. Ralph for helpful discussions and comments.

\section{REFERENCES}

1) R. W. Hertzberg: Deformation and Fracture Mechanisms of Engineer- ing Materials, (John Wiley \& Sons, 1996) pp. 452.

2) L. L. Shaw: Journal of Metals (JOM) 52 (2000) 41-45.

3) N. Tsuji, Y. Saito, S. H. Lee and Y. Minamino: Adv. Eng. Mater. 5 (2003) 338-344.

4) Severe Plastic Deformation: Towards Bulk Production of Nanostructured Materials, ed. by B. S. Altan, (Nova Science Publishers, 2005).

5) R. Z. Abdulov, R. Z. Valiev and N. A. Krasilnikov: J. Mater. Sci. Lett. 9 (1990) 1445-1447.

6) V. M. Segal: Mater. Sci. Eng. A 197 (1995) 157-164.

7) Y. Iwahashi, J. Wang, Z. Horita, M. Nemoto and T. G. Langdon: Scr. Mater. 35 (1996) 143-146.

8) J. Richert and M. Richert: Aluminium 62 (1986) 604-607.

9) Y. Saito, H. Utsunomiya, N. Tsuji and T. Sakai: Acta Mater. 47 (1999) 579-583.

10) N. Tsuji, N. Kamikawa, H. W. Kim and Y. Minamino: Ultrafine Grained Materials III, ed. by Y. T. Zhu, T. G. Langdon, R. Z. Valiev, S. L. Semiatin, D. H. Shin and T. C. Lowe, (TMS, 2004) pp. 219-226.

11) N. Tsuji, Y. Ito, Y. Saito and Y. Minamino: Scr. Mater. 47 (2002) 893899.

12) N. Tsuji, X. Huang and H. Nakashima: Proc. of 25th Risø Int. Symp. on Mater. Sci.: Evolution of Deformation Microstructures in 3D, (Ris $\emptyset$ National Laboratory, Roskilde, Denmark, 2004) pp. 147-170.

13) N. Kamikawa, N. Tsuji, X. Huang and N. Hansen: Acta Mater. 54 (2006) 3055-3066.

14) Q. Liu: J. App. Cryst. 27 (1994) 755-761.

15) F. J. Humphreys, Y. Huang, I. Brough and C. Harris: J. Microscopy 195 (1999) 212-216.

16) F. J. Humphreys: J. Mater. Sci. 36 (2001) 3833-3854.

17) J. Grewen and J. Huber: Recrystallization of Metallic Materials, ed. by F. Haessner, (Dr. Riederer Verlag GmbH, 1978) pp. 111.

18) J. Hirsch and K. Lücke: Textures and Microstructures, $8 \& 9$ (1988) $131-151$.

19) J. Hirsch and K. Lücke: Acta Metall. 33 (1985) 1927-1938.

20) J. Hirsch and K. Lücke: Acta Metall. 36 (1988) 2863-2882.

21) I. L. Dillamore and W. T. Roberts: J. Inst. Metals 92 (1963-64) 193199.

22) W. Truszkowski, J. Król and B. Major: Metall. Trans. A 11A (1980) 749-758.

23) W. Truszkowski, J. Król and B. Major: Metall. Trans. A 13A (1982) 665-669.

24) X. Huang, N. Kamikawa, N. Tsuji and N. Hansen: Ultrafine Grained Materials $I V$, ed. by Y. T. Zhu, T. G. Langdon, Z. Horita, M. J. Zehetbauer, S. L. Semiatin and T. C. Lowe, (TMS, 2006) pp. 307-314.

25) C. P. Heason and P. B. Prangnell: Proc. Conf. "Nanomaterials by Severe Plastic Deformation-NanoSPD2," ed. by Zehetbauer M, Valiev RZ, (2002) pp. 498-504.

26) C. P. Heason and P. B. Prangnell: Mater. Sci. Forum 408-412 (2002) 733-738.

27) B. L. Li, N. Tsuji and N. Kamikawa: Mater. Sci. Eng. A 423 (2006) 331-342.

28) N. Kamikawa, N. Tsuji and Y. Minamino: Sci. Technol. Adv. Mater. 5 (2004) 163-172.

29) T. Sakai, Y. Saito and K. Kato: Trans. ISIJ 27 (1987) 520-525.

30) T. Sakai, Y. Saito, M. Matsuo and K. Kawasaki: ISIJ Int. 31 (1991) 8694.

31) S. H. Lee, Y. Saito, T. Sakai and H. Utsunomiya: Mater. Sci. Eng. A 325 (2002) 228-235.

32) H. W. Kim, S. B. Kang, Z. P. Xing, N. Tsuji and Y. Minamino: Mater. Sci. Forum 408-412 (2002) 727-732.

33) T. Sakai, Y. Saito, K. Hirano and K. Kato: Trans. ISIJ 28 (1988) 10281035.

34) I. Ya. Tarnovski, A. A. Pozdeyev and V. B. Lyashikov: Deformation of Metals during Rolling, (Pergamon Press, 1965).

35) Y. Saito et al.: Metal forming, (The Japan Institute of Metals (JIM), 1986).

36) O. V. Mishin, B. Bay and D. Juul Jensen: Metall. Mater. Trans. A 31A (2000) 1653-1662.

37) H. O. Asbeck and H. Mecking: Mater. Sci. Eng. 34 (1978) 111-119. 